01,07

\title{
Электронные и механические свойства, фазовая стабильность и энергии образования точечных дефектов боронитрида $\mathrm{Nb}_{2} \mathrm{BN}$
}

\author{
(С Д.В. Суетин, И.Р. Шеин \\ Институт химии твердого тела УрО РАН, \\ Екатеринбург, Россия \\ E-mail: suetin@ihim.uran.ru
}

(Поступила в Редакцию 24 января 2017 г.)

Ab initio полнопотенциальным методом FLAPW-GGA установлены электронная структура, поверхность Ферми, коэффициенты Зоммерфельда и парамагнитной восприимчивости Паули, энергии когезии, формирования фаз и точечных дефектов, константы упругости, модули сжатия, сдвига и Юнга, коэффициенты Пуассона, микротвердость по Виккерсу боронитрида $\mathrm{Nb}_{2} \mathrm{BN}$, которые обсуждаются в сравнении с аналогичными данными для борокарбида $\mathrm{Mo}_{2} \mathrm{BC}$ и других родственных бинарных карбидов, нитридов и боридов переходных металлов, а также с имеющимися экспериментальными данными.

DOI: 10.21883/FTT.2017.08.44743.13

\section{1. Введение}

Карбиды и нитриды переходных металлов обладают комплексом уникальных механических, электрических и термических свойств (высокие твердость, износостойкость, тугоплавкость, сверхпроводимость). Вследствие этого чрезвычайно высока технологическая значимость данных материалов: они используются при изготовлении износостойких покрытий на режущих инструментах, высокотемпературных конструкционных материалов, в электрических схемах и др. [1-3]. С другой стороны, бориды переходных металлов обладают набором перспективных физико-химических характеристик и находят применение в качестве компонентов тугоплавких сплавов, высокопрочных защитных покрытий, основы твердых припоев, абразивных и коррозионностойких материалов [3-6].

Наряду с бинарными карбидами, нитридами и боридами переходных металлов в настоящее время широко применяются и многокомпонентные системы на их основе, в том числе карбонитриды, борокарбиды, боронитриды, которые могут представлять собой твердые растворы замещения или обладать индивидуальной кристаллической структурой и свойствами $[1,2,4]$. Для использования соединения в качестве износостойкого покрытия оно должно обладать высокой жесткостью совместно с достаточной пластичностью. Недавно для этой цели был предложен борокарбид молибдена $\mathrm{Mo}_{2} \mathrm{BC}$ с орторомбической структурой, родственный так называемым наноламинатам или МАХ-фазам [7,8]. Синтез $\mathrm{Mo}_{2} \mathrm{BC}$ осуществлялся посредством дуговой плавки в атмосфере $\operatorname{Ar}[9,10]$, а также горячим прессованием механической смеси чистых Мо, В и С [11]. Кроме того, повышенное внимание исследователей к $\mathrm{Mo}_{2} \mathrm{BC}$ было вызвано обнаружением у него сверхпроводящего перехода $\left(T_{C} \sim 7 \mathrm{~K}\right)[10,11]$.

В дальнейшем был синтезирован (из смеси металлического ниобия и нитрида бора) боронитрид ниобия
$\mathrm{Nb}_{2} \mathrm{BN}$ [12], имеющий тот же тип кристаллической структуры, что и $\mathrm{Mo}_{2} \mathrm{BC}$, для которого также была обнаружена сверхпроводимость $\left(T_{C} \sim 2.2 \mathrm{~K}\right)$ при неопределенном нестехиометрическом составе $\mathrm{Nb}_{2} \mathrm{BN}_{1-x}$. В недавней работе [13] с помощью твердофазного синтеза (также из чистого металла и нитрида бора) были получены поликристаллические образцы $\mathrm{Nb}_{2} \mathrm{BN}$, для которых была подтверждена сверхпроводимость при $T_{C} \sim 4.4 \mathrm{~K}$.

Современные высокопроизводительные теоретические расчетные методы, основанные на теории функционала электронной плотности (DFT), позволяют предсказать большой набор физико-химических параметров исследуемых систем, определить потенциальную возможность их практического применения. Вместе с тем в отличие от $\mathrm{Mo}_{2} \mathrm{BC}$ и родственных борокарбидов, для которых были проведены расчеты структурных, электронных, механических свойств, сделаны оценки энергетической стабильности $[7,8]$, аналогичные сведения о $\mathrm{Nb}_{2} \mathrm{BN}$ крайне ограничены.

В настоящей работе с использованием полнопотенциального линеаризованного метода присоединенных плоских волн с обобщенной градиентной аппроксимацией обменно-корреляционного потенциала (FLAPW-GGA) нами впервые изучены электронная структура, механические свойства (независимые константы упругости, модули сжатия, сдвига и Юнга, отношения Пуассона, оценки микротвердости по Виккерсу), энергии образования фаз и когезии, энергии образования вакансий, межатомные взаимодействия в боронитриде $\mathrm{Nb}_{2} \mathrm{BN}$ в сравнении с данными для изоструктурного борокарбида $\mathrm{Mo}_{2} \mathrm{BC}$.

\section{2. Модели и метод расчетов}

Боронитрид $\mathrm{Nb}_{2} \mathrm{BN}$ обладает орторомбической структурой типа $\mathrm{Mo}_{2} \mathrm{BC}$ (пространственная группа $\mathrm{Cmcm}$ (\#63)) и при этом является одним из немногих чисто 


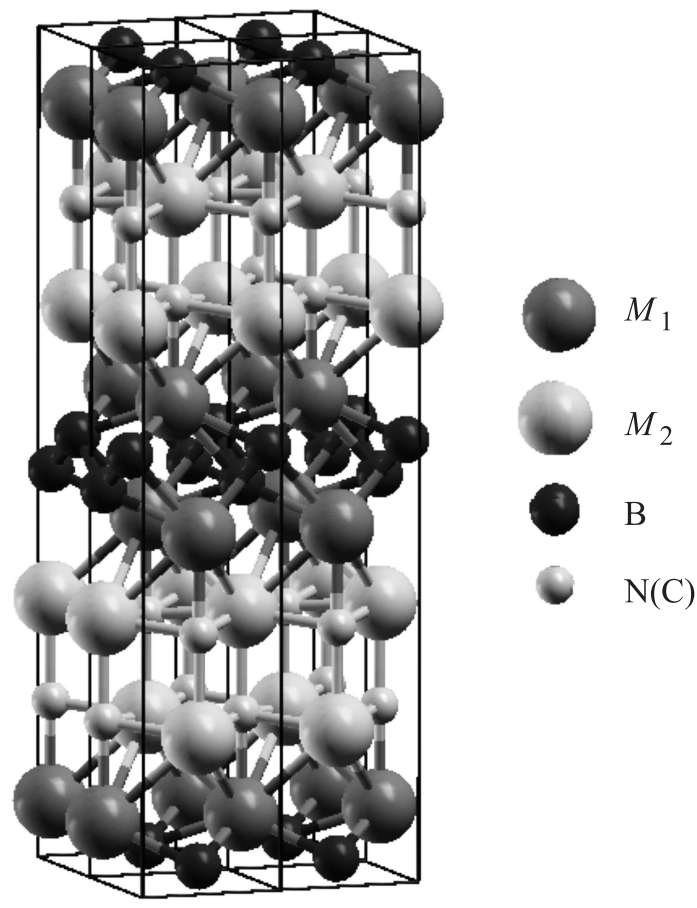

Рис. 1. Фрагмент модельной кристаллической структуры нового боронитрида $\mathrm{Nb}_{2} \mathrm{BN}$ (структурный тип $\mathrm{Mo}_{2} \mathrm{BC}$ ), использованный в расчетах FLAPW-GGA.

тройных соединений, в которых содержатся атомы переходного металла, бора и азота (углерода) [1]. В этих соединениях атомы азота (углерода) расположены в октаэдрах $\left[M_{6} X\right]$ (где $\left.M=\mathrm{Nb}, \mathrm{Mo} ; X=\mathrm{C}, \mathrm{N}\right)$, которые сочленяются между собой ребрами и образуют слои, отделенные друг от друга гофрированными сетками атомов бора (рис. 1). Последние проходят через тригональные призмы, образованные атомами $\mathrm{Nb}(\mathrm{Mo})$, что является типичным для моноборидов переходных металлов. В такой кристаллической структуре атомы металла занимают две неэквивалентные позиции: $M_{1} 4 c$ $\left(0 ; y_{1} ; 0.25\right)$ и $M_{2} 4 c\left(0 ; y_{2} ; 0.25\right)$. Атомы бора расположены в позициях В $4 c\left(0 ; y_{3} ; 0.25\right)$, а атомы азота и углерода в позициях $4 c\left(0 ; y_{4} ; 0.25\right)$.

Расчеты были проведены с использованием полнопотенциального линеаризованного метода присоединенных плоских волн (FLAPW), код WIEN2k [14]. Для обменно-корреляционного потенциала была выбрана обобщенная градиентная аппроксимация (GGA) в форме PBE (Perdew-Burke-Ernzerhof) [15]. В качестве стартовых брались следующие электронные конфигурации атомов: для ниобия - $[\mathrm{Kr}] 5 s^{2} 4 d^{3}$, для молибдена $[\mathrm{Kr}] 5 s^{2} 4 d^{4}$, для бора $-[\mathrm{He}] 2 s^{2} 2 p^{1}$, для углерода $[\mathrm{He}] 2 s^{2} 2 p^{2}$, для азота - $[\mathrm{He}] 2 s^{2} 2 p^{3}$. Радиусы атомных MT (muffin-tin)-сфер составляли 2.00 a.u. (Mo, Nb), 1.65 a.u. (B, C, N).

Релятивистские эффекты учитывались в рамках скалярно-релятивистского приближения. Валентная часть волновой функции была взята с потенциалом, разло- женным по сферическим гармоникам до $l=4$. Внутри атомных сфер волновые функции были разложены в ряд вплоть до $l=12$. Параметр плосковолнового разложения $R_{\mathrm{MT}} K_{\max }$ был выбран равным 7.0 при полном числе $k$-точек в зоне Бриллюэна $10 \times 10 \times 10$. В качестве двух критериев сходимости $a b$ initio зонных FLAPW-расчетов использовались разность энергий между последовательными итерациями $\Delta E<0.01 \mathrm{mRy}$, а также величины сил, действующих на атомы $f_{i}<1 \mathrm{mRy} / \mathrm{a} . \mathrm{u}$. Плотность электронных состояний была получена с использованием модифицированного метода тетраэдров [16].

Для боронитрида $\mathrm{Nb}_{2} \mathrm{BN}$ и борокарбида $\mathrm{Mo}_{2} \mathrm{BC}$ расчеты были проведены в спин-ограниченном и спин-поляризованном (в приближении ферромагнитного упорядочения) базисах, при этом магнитное решение сводилось к немагнитному, т. е. к отсутствию локальных магнитных моментов на атомах.

\section{3. Результаты и обсуждение}

На первом этапе путем минимизации полной энергии систем $E_{\text {tot }}$ определялись оптимальные параметры кристаллических решеток $a, b, c$, а также атомные позиции и межатомные расстояния. Результаты представлены в табл. 1-3. Можно видеть, что полученные данные для боронитрида $\mathrm{Nb}_{2} \mathrm{BN}$ и борокарбида $\mathrm{Mo}_{2} \mathrm{BC}$ полностью соответствуют имеющимся экспериментальным результатам $[1,12]$, а также теоретическим расчетам $[7,8]$. Кроме того, из значения $V$ была рассчитана теоретическая плотность $\rho$ для $\mathrm{Nb}_{2} \mathrm{BN}$ и $\mathrm{Mo}_{2} \mathrm{BC}$ (табл. 1 ).

Фазовая устойчивость $\mathrm{Nb}_{2} \mathrm{BN}$ и $\mathrm{Mo}_{2} \mathrm{BC}$ была определена из расчетов их энергий формирования $E_{\text {form }}$ относительно наиболее стабильных из полиморфных модификаций составляющих соединение элементов. Так, например, энергия формирования боронитрида $\mathrm{Nb}_{2} \mathrm{BN}$ рассчитывалась как $E_{\text {form }}\left(\mathrm{Nb}_{2} \mathrm{BN}=E_{\mathrm{tot}}\left(\mathrm{Nb}_{2} \mathrm{BN}\right)\right.$ $-\left[2 E_{\mathrm{tot}}(\mathrm{Nb})+(1 / 12) E_{\mathrm{tot}}\left(\alpha-\mathrm{B}_{12}\right)+(1 / 2) E_{\mathrm{tot}}\left(\mathrm{N}_{2}\right)\right]$, где $E_{\text {tot }}$ - полные энергии оптимизированных структур боронитрида $\mathrm{Nb}_{2} \mathrm{BN}$, металлического ниобия, кристаллического $\alpha$-В 12 и молекулы азота, полученные из зонных расчетов FLAPW-GGA. Аналогично энергия формирования $\mathrm{Mo}_{2} \mathrm{BC}$ была получена как $E_{\text {form }}\left(\mathrm{Mo}_{2} \mathrm{BC}\right)=E_{\text {tot }}\left(\mathrm{Mo}_{2} \mathrm{BC}\right)-\left[2 E_{\text {tot }}(\mathrm{Mo})\right.$ $\left.+(1 / 12) E_{\text {tot }}\left(\alpha-\mathrm{B}_{12}\right)+E_{\text {tot }}\left(\mathrm{C}_{g}\right)\right]$, где $E_{\text {tot }}-$ полные энергии борокарбида $\mathrm{Mo}_{2} \mathrm{BC}$, металлического молибдена, $\alpha-\mathrm{B}_{12}$ и графита $\mathrm{C}^{g}$. Согласно данному подходу, соединение считается термодинамически стабильным в том случае, если $E_{\text {form }}<0$. Напротив, когда $E_{\text {form }}>0$, соединение является нестабильным, и его получение при нормальных условиях будет проблематичным. Рассчитанные для $\mathrm{Nb}_{2} \mathrm{BN}$ и $\mathrm{Mo}_{2} \mathrm{BC}$ значения $E_{\text {form }}$ представлены в табл. 4. Можно видеть, что и борокарбид, и боронитрид обладают энергетической стабильностью, так как значения $E_{\text {form }}<0$, при этом $\mathrm{Nb}_{2} \mathrm{BN}$ получается более стабильным, чем $\mathrm{Mo}_{2} \mathrm{BC}$, что совпадает с оценками для стабильных моноборидов $\mathrm{NbB}$ и $\mathrm{MoB}\left(E_{\text {form }}=-0.81\right.$ 
Таблица 1. Оптимизированные параметры решетки $a, b, c$, объемы элементарной ячейки $V$ и теоретическая плотность $\rho$ для боронитрида $\mathrm{Nb}_{2} \mathrm{BN}$ и борокарбида $\mathrm{Mo}_{2} \mathrm{BC}$, полученные из расчетов FLAPW-GGA

\begin{tabular}{c|c|c|c|c|c}
\hline Система & $a, \mathrm{~nm}$ & $b, \mathrm{~nm}$ & $c, \mathrm{~nm}$ & $V, \mathrm{~nm}^{3}$ & $\rho, \mathrm{kg} / \mathrm{m}^{3}$ \\
\hline $\mathrm{Nb}_{2} \mathrm{BN}$ & 0.31721 & 1.79750 & 0.31249 & 0.089089 & 7852 \\
& $(0.317[13])$ & $(1.785[13])$ & $(0.311[13])$ & $(0.087989[13])$ & 8535 \\
$\mathrm{Mo}_{2} \mathrm{BC}$ & 0.31144 & 1.75022 & 0.30652 & 0.083538 & $(0.081571[9]$, \\
& $(0.3086[9]$, & $(1.735[9]$, & $(0.3047[9]$, & $0.084962[7]$, & \\
& $0.3094[7]$, & $1.7768[7]$, & $0.3091[7]$, & $0.084496[8])$
\end{tabular}

Примечан ие. В скобках приводятся имеющиеся экспериментальные $[9,13]$ и теоретические $[7,8]$ данные.

Таблица 2. Оптимизированные атомные позиции $(x ; y ; z)$ для исследуемого боронитрида $\mathrm{Nb}_{2} \mathrm{BN}$, а также борокарбида Мо $2 \mathrm{BC}$

\begin{tabular}{c|c|c|c|c}
\hline Система & $M_{1}$ & $M_{2}$ & $\mathrm{~N}(\mathrm{C})$ & $\mathrm{B}$ \\
\hline $\mathrm{Nb}_{2} \mathrm{BN}$ & $(0 ; 0.07281 ; 0.25)$ & $(0 ; 0.31784 ; 0.25)$ & $(0 ; 0.19410 ; 0.25)$ & $(0 ; 0.47303 ; 0.25)$ \\
& $(0 ; 0.0721 ; 0.25[13])$ & $(0 ; 0.3139 ; 0.25[13])$ & $(0 ; 0.192 ; 0.25[13])$ & $(0 ; 0.4731 ; 0.25[13])$ \\
$\mathrm{Mo}_{2} \mathrm{BC}$ & $(0 ; 0.07174 ; 0.25)$ & $(0 ; 0.31445 ; 0.25)$ & $(0 ; 0.19117 ; 0.25)$ & $(0 ; 0.47193 ; 0.25)$ \\
& $(0 ; 0.0705 ; 0.25[9])$ & $(0 ; 0.314 ; 0.25[9])$ & $(0 ; 0.192 ; 0.25[9])$ & $(0 ; 0.473 ; 0.25[9])$
\end{tabular}

Пр имечан ие. В скобках приводятся имеющиеся экспериментальные данные $[9,13]$.

Таблица 3. Рассчитанные межатомные расстояния $d^{X-Y}$ (в $\mathrm{nm}$ ) для исследуемого боронитрида $\mathrm{Nb}_{2} \mathrm{BN}$ и борокарбида $\mathrm{Mo}_{2} \mathrm{BC}$

\begin{tabular}{|c|c|c|c|c|c|c|}
\hline Система & $d^{M_{1}-\mathrm{N}(\mathrm{C})}$ & $d^{M_{2}-\mathrm{N}(\mathrm{C})}$ & $d^{M_{1}-\mathrm{B}}$ & $d^{M_{1}-M_{1}}$ & $d^{M_{1}-M_{2}}$ & $d^{\mathrm{B}-\mathrm{B}}$ \\
\hline $\mathrm{Nb}_{2} \mathrm{BN}$ & 0.21803 & $\begin{array}{l}0.22242 \\
0.22369\end{array}$ & 0.23741 & 0.30483 & 0.29700 & 0.18389 \\
\hline $\mathrm{Mo}_{2} \mathrm{BC}$ & 0.20903 & $\begin{array}{l}0.21577 \\
0.21871\end{array}$ & 0.23147 & 0.29419 & 0.29566 & 0.18205 \\
\hline
\end{tabular}

и $-0.52 \mathrm{eV} /$ atom соответственно) с орторомбической структурой типа $\mathrm{CrB}$ [17]. Сделанный вывод об устойчивости $\mathrm{Mo}_{2} \mathrm{BC}$ также подтверждается оценкой соответствующей $E_{\text {form}}$, сделанной в работе [8], в которой борокарбид рассчитывался методом VASP-GGA.

Кроме того, помимо энергий формирования для оценки стабильности $\mathrm{Nb}_{2} \mathrm{BN}$ и $\mathrm{Mo}_{2} \mathrm{BC}$ были рассчитаны также их энергии когезии $E_{\text {coh. }}$ В частности, для боронитрида $\mathrm{Nb}_{2} \mathrm{BN} E_{\text {соһ }}$ вычислялась как $E_{\mathrm{coh}}\left(\mathrm{Nb}_{2} \mathrm{BN}\right)=$ $=E_{\mathrm{tot}}\left(\mathrm{Nb}_{2} \mathrm{BN}\right)-\left[2 E_{\mathrm{at}}(\mathrm{Nb})+E_{\mathrm{at}}(\mathrm{B})+E_{\mathrm{at}}(\mathrm{N})\right]$, где $E_{\mathrm{at}}-$

Таблица 4. Энергии формирования $E_{\text {form }}$ из простых элементов, энергии когезии $E_{\text {coh }}$ и энергии образования вакансий по подрешеткам $\mathrm{N}(\mathrm{C})$ и В $E_{f v}$ при концентрации вакансий $x=0.0625$ для $\mathrm{Nb}_{2} \mathrm{BN}$ и $\mathrm{Mo}_{2} \mathrm{BC}$

\begin{tabular}{c|c|c|c|c}
\hline \multirow{2}{*}{ Система } & \multirow{2}{*}{$E_{\text {form }}, \mathrm{eV} /$ atom } & \multirow{2}{*}{$E_{\text {coh }}, \mathrm{eV} / \mathrm{atom}$} & \multicolumn{2}{|c}{$E_{f v}, \mathrm{eV}$} \\
\cline { 4 - 5 } & & & $\mathrm{N}(\mathrm{C})$ & \multicolumn{1}{c}{$\mathrm{B}$} \\
\hline $\mathrm{Nb}_{2} \mathrm{BN}$ & -1.06 & -9.60 & -0.47 & -0.01 \\
$\mathrm{Mo}_{2} \mathrm{BC}$ & $-0.42(-0.283)$ & -9.70 & -0.30 & 1.12
\end{tabular}

Примечание. В скобках приведены данные расчетной работы [8]. полные энергии изолированных атомов ниобия, бора и азота. Определенная таким способом $E_{\text {coh характеризует }}$ межатомные взаимодействия и по физическому смыслу составляет полную энергию, которую необходимо затратить для того, чтобы разделить кристалл на отдельные атомы. Результаты проведенных расчетов суммированы в табл. 4. Видно, что значения $E_{\text {coh }}$ для $\mathrm{Nb}_{2} \mathrm{BN}$ и $\mathrm{Mo}_{2} \mathrm{BC}$ сравнимы по величине. Это указывает на тождественность природы межатомных взаимодействий в этих системах.

Многие карбиды и нитриды переходных металлов обладают значительной нестехиометрией по неметаллической подрешетке [18]. Кроме того, изучаемый боронитрид $\mathrm{Nb}_{2} \mathrm{BN}$ получен с неизвестной нестехиометрией по азоту [12,13], поэтому вызывает интерес определение возможности образования вакансий в такой системе. Для установления вероятности существования таких структурных вакансий в $\mathrm{Nb}_{2} \mathrm{BN}$ и $\mathrm{Mo}_{2} \mathrm{BC}$ были рассчитаны их энергии образования $E_{f v}$ по подрешеткам $\mathrm{N}(\mathrm{C})$ и В для случая $x=0.0625$. Для этого был использован метод сверхъячеек, из которых удалялся один из атомов неметалла, при этом проводилась полная минимизация энергии сверхъячеек (с учетом параметров их решеток и позиций атомов). В нашем случае формальный со- 
Таблица 5. Рассчитанные константы упругости $C_{i j}$ (в $\mathrm{GPa}$ ) для боронитрида $\mathrm{Nb}_{2} \mathrm{BN}$ и борокарбида $\mathrm{Mo}_{2} \mathrm{BC}$

\begin{tabular}{c|c|c|c|c|c|c|c|c|c}
\hline Система & $C_{11}$ & $C_{22}$ & $C_{33}$ & $C_{12}$ & $C_{13}$ & $C_{23}$ & $C_{44}$ & $C_{55}$ & $C_{66}$ \\
\hline $\mathrm{Nb}_{2} \mathrm{BN}$ & 485.1 & 564.3 & 513.4 & 156.2 & 194.6 & 139.6 & 174.4 & 207.0 & 178.6 \\
$\mathrm{Mo}_{2} \mathrm{BC}$ & 523.0 & 640.3 & 559.1 & 214.4 & 221.0 & 206.6 & 176.8 & 277.0 & 187.9 \\
& $(551[7])$ & $(566[7])$ & $(553[7])$ & $(211[7])$ & $(204[7])$ & $(210[7])$ & $(168[7])$ & $(241[7])$ & $(182[7])$
\end{tabular}

Примечание. В скобках приводятся результаты теоретических расчетов [7].

став нестехиометрических фаз составлял $M_{2} \mathrm{~B} X_{0.9375}$ и $M_{2} \mathrm{~B}_{0.9375} X$, где $M=\mathrm{Nb}, \mathrm{Mo}, X=\mathrm{C}, \mathrm{N}$ (или сверхъячейки с содержанием атомов $\left.M_{32} \mathrm{~B}_{15} X_{16}\left(M_{32} \mathrm{~B}_{16} X_{15}\right)\right)$.

Как указывалось выше, синтез $\mathrm{Nb}_{2} \mathrm{BN}$ и $\mathrm{Mo}_{2} \mathrm{BC}$ был осуществлен твердофазным способом. Поэтому для определения энергий образования вакансий по подрешеткам бора, азота и углерода нами была использована общепринятая методика расчета этих энергий через химический потенциал [19]

$$
E_{f v}=E_{\mathrm{tot}}\left(M_{2} \mathrm{~B}_{y} X_{z}\right)-E_{\mathrm{tot}}\left(M_{2} \mathrm{~B} X\right)-\sum_{i} n_{i} \mu_{i},
$$

где $E_{f v}$ - энергия образования незаряженной вакансии, $E_{\text {tot }}\left(M_{2} \mathrm{~B} X\right)$ и $E_{\text {tot }}\left(M_{2} \mathrm{~B}_{y} X_{z}\right)-$ энергии идеальной и дефектной решеток соответственно, $n_{i}$ и $\mu_{i}$ - концентрация вакансий по подрешетке $i$ и ее химический потенциал соответственно. Для расчета энергий образования вакансий в $\mathrm{Nb}_{2} \mathrm{BN}$ были использованы химические потенциалы бора и азота, определенные через химические потенциалы для кристаллического нитрида бора

$$
\mu_{\mathrm{B}}+\mu_{\mathrm{N}}=E(\mathrm{BN}),
$$

где $\mu_{\mathrm{B}}$ и $\mu_{\mathrm{N}}$ - химические потенциалы бора и азота для кристаллического нитрида бора, $E(\mathrm{BN})$ - энергия, приходящаяся на пару $\mathrm{B}-\mathrm{N}$ в кристаллическом состоянии. Химический потенциал бора $\mu_{\mathrm{B}}$ в наших расчетах выбирался как энергия, приходящаяся на один атом бора в кристаллической структуре $\alpha-\mathrm{B}_{12}$, а химический потенциал азота - как разность

$$
\mu_{\mathrm{N}}=E(\mathrm{BN})-\mu_{\mathrm{B}}
$$

Для расчета энергий образования вакансий по подрешеткам бора и углерода в $\mathrm{Mo}_{2} \mathrm{BC}$ химический потенциал $\mu_{\mathrm{B}}$ был выбран так же как энергия, приходящаяся на один атом бора в структуре $\alpha$ - $\mathrm{B}_{12}$, а химический потенциал $\mu_{\mathrm{C}}$ был равен энергии, приходящейся на один атом углерода для структуры графита.

Полученные результаты приведены в табл. 4. Видно, что для $\mathrm{Nb}_{2} \mathrm{BN}$ и $\mathrm{Mo}_{2} \mathrm{BC}$ значения энергий образования вакансий по подрешеткам азота и углерода имеют отрицательные значения, что позволяет сделать вывод об энергетической выгодности такой нестехиометрии в данных соединениях. Это утверждение полностью совпадает с экспериментальными данными [13], согласно которым кристаллическая структура $\mathrm{Nb}_{2} \mathrm{BN}$ определена как нестехиометрическая по азоту. Что касается энергий образования вакансий по подрешетке бора, то, как видно из табл. 4, их значения отрицательны, но крайне малы по абсолютной величине (для $\mathrm{Nb}_{2} \mathrm{BN}$ ) или положительны (для $\mathrm{Mo}_{2} \mathrm{BC}$ ). Этот факт позволяет сделать вывод о том, что образование вакансий по подрешетке бора в этих структурах будет маловероятным.

Далее обсудим механические свойства $\mathrm{Nb}_{2} \mathrm{BN}$ и $\mathrm{Mo}_{2} \mathrm{BC}$, полученные нами в результате проведенных расчетов FLAPW-GGA при использовании тензора напряжений при различных искажениях, примененных к равновесной кристаллической структуре для получения девяти независимых констант упругости $C_{i j}$, значения которых приведены в табл. 5. Все полученные $C_{i j}$ для $\mathrm{Nb}_{2} \mathrm{BN}$ и $\mathrm{Mo}_{2} \mathrm{BC}$ положительны и удовлетворяют общему критерию механической стабильности кристаллов орторомбической симметрии: $C_{11}>0, C_{22}>0, C_{33}>0$, $C_{44}>0, C_{55}>0, C_{66}>0, C_{11}+C_{22}+C_{33}+2\left(C_{12}+C_{13}\right.$ $\left.+C_{23}\right)>0,\left(C_{11}+C_{22}-2 C_{12}\right)>0,\left(C_{11}+C_{33}-2 C_{13}\right)>0$ и $\left(C_{22}+C_{33}-2 C_{23}\right)>0[20]$.

Затем с использованием коэффициентов $C_{i j}$ были рассчитаны модули сжатия $B$ и модули сдвига $G$ для моно- и поликристаллических образцов $\mathrm{Nb}_{2} \mathrm{BN}$, $\mathrm{Mo}_{2} \mathrm{BC}$ в аппроксимациях Фойгта [21], Реусса [22] и Фойгта-Реусса-Хилла [23] как

$$
\begin{aligned}
& B_{\mathrm{V}}=(1 / 9)\left[C_{11}+C_{22}+C_{33}+2\left(C_{12}+C_{13}+C_{23}\right)\right], \\
& G_{\mathrm{V}}=(1 / 15)\left[C_{11}+C_{22}+C_{33}\right. \\
&\left.+3\left(C_{44}+C_{55}+C_{66}\right)-\left(C_{12}+C_{13}+C_{23}\right)\right], \\
& B_{\mathrm{R}}=\Delta\left[C_{11}\left(C_{22}+C_{33}-2 C_{23}\right)+C_{22}\left(C_{33}-2 C_{13}\right)\right. \\
& \quad-2 C_{33} C_{12}+C_{12}\left(2 C_{23}-C_{12}\right) \\
&\left.+C_{13}\left(2 C_{12}-C_{13}\right)+C_{23}\left(2 C_{13}-C_{23}\right)\right]^{-1}, \\
& G_{\mathrm{R}}=15\left\{4 \left[C_{11}\left(C_{22}+C_{33}+C_{23}\right)\right.\right. \\
&+C_{22}\left(C_{33}+C_{13}\right)+C_{33} C_{12}-C_{12}\left(C_{23}+C_{12}\right) \\
&\left.\quad-C_{13}\left(C_{12}+C_{13}\right)-C_{23}\left(C_{13}+C_{23}\right)\right] / \Delta \\
&\left.+3\left[\left(1 / C_{44}+1 / C_{55}+1 / C_{66}\right)\right]\right\} \\
& \quad C_{13}\left(C_{12} C_{23}-C_{13} C_{22}\right)+C_{23}\left(C_{12} C_{13}-C_{23} C_{11}\right) \\
&+ C_{33}\left(C_{11} C_{22}-\left(C_{12}\right)^{2}\right), \\
& B_{\mathrm{VRH}}=(1 / 2)\left(B_{\mathrm{R}}+B_{\mathrm{V}}\right), \\
& \quad G_{\mathrm{VRH}}=(1 / 2)\left(G_{\mathrm{R}}+G_{\mathrm{V}}\right)(\mathrm{cm} .[20]) .
\end{aligned}
$$


Таблица 6. Рассчитанные модули сжатия $B$ (в GPa) и модули сдвига $G$ (в GPa), их отношение $B_{\mathrm{VRH}} / G_{\mathrm{VRH}}$, сжимаемость $\beta$ $\left(\right.$ в $\left.\mathrm{GPa}^{-1}\right)$, модули Юнга $Y$ (в GPa), отношение Пуассона $v$ и коэффициенты Ламе $\mu, \lambda$ (в $\left.\mathrm{GPa}\right)$ для нового боронитрида $\mathrm{Nb}_{2} \mathrm{BN}$ и борокарбида $\mathrm{Mo}_{2} \mathrm{BC}$

\begin{tabular}{|c|c|c|c|c|c|c|}
\hline Система & $B_{\mathrm{V}}$ & $B_{\mathrm{R}}$ & $B_{\mathrm{VRH}}$ & $G_{\mathrm{V}}$ & $G_{\mathrm{R}}$ & $G_{\mathrm{VRH}}$ \\
\hline $\mathrm{Nb}_{2} \mathrm{BN}$ & 282.6 & 282.5 & 282.6 & 183.5 & 180.9 & 182.2 \\
\hline $\mathrm{Mo}_{2} \mathrm{BC}$ & $\begin{array}{c}334.0 \\
(324[7])\end{array}$ & $\begin{array}{c}332.4 \\
(324[7])\end{array}$ & $\begin{array}{c}333.2 \\
(324[7], \\
313[8])\end{array}$ & $\begin{array}{c}200.4 \\
188[7]\end{array}$ & $\begin{array}{c}193.2 \\
(185[7])\end{array}$ & $\begin{array}{c}196.8 \\
(187[7], \\
191[8])\end{array}$ \\
\hline Система & $\beta$ & $B_{\mathrm{VRH}} / G_{\mathrm{VRH}}$ & $\gamma$ & $v$ & $\mu$ & $\lambda$ \\
\hline $\mathrm{Nb}_{2} \mathrm{BN}$ & 0.00354 & 1.55 & 449.9 & 0.235 & 182.2 & 161.1 \\
\hline $\mathrm{Mo}_{2} \mathrm{BC}$ & 0.00300 & $\begin{array}{c}1.69 \\
(1.73[8])\end{array}$ & $\begin{array}{c}493.2 \\
(470[7], \\
455[8])\end{array}$ & $\begin{array}{c}0.253 \\
(0.26[7])\end{array}$ & 196.8 & 202.0 \\
\hline
\end{tabular}

Примечание. В скобках приведены результаты теоретических расчетов $[7,8]$.

Кроме того, мы также рассчитали такие механические параметры, как сжимаемость $\beta=1 / B_{\mathrm{VRH}}$, модули Юнга

$$
Y=\frac{9 B_{\mathrm{VRH}} G_{\mathrm{VRH}}}{3 B_{\mathrm{VRH}}+G_{\mathrm{VRH}}}
$$

и отношение Пуассона

$$
v=\frac{3 B_{\mathrm{VRH}}-2 G_{\mathrm{VRH}}}{2\left(3 B_{\mathrm{VRH}}+G_{\mathrm{VRH}}\right)},
$$

а также коэффициенты Ламе

$$
\mu=\frac{Y}{2(1+v)}, \quad \lambda=\frac{v Y}{(1+v)(1-2 v)} .
$$

Вычисленные механические параметры для $\mathrm{Nb}_{2} \mathrm{BN}$ и $\mathrm{Mo}_{2} \mathrm{BC}$ приведены в табл. 6. Видно, что рассчитанные значения упругих модулей, отношения Пуассона в случае $\mathrm{Mo}_{2} \mathrm{BC}$ совпадают с полученными в теоретических работах $[7,8]$. Модули сжатия $B$, модули сдвига $G$ и модули Юнга $Y$ в случае $\mathrm{Mo}_{2} \mathrm{BC}$ на $10-20 \%$ выше соответствующих значений для $\mathrm{Nb}_{2} \mathrm{BN}$, это свидетельствует о более высокой твердости борокарбида. Напротив, большей сжимаемостью $\beta=1 / B$ обладает $\mathrm{Nb}_{2} \mathrm{BN}$. Необходимо подчеркнуть, что для этих систем выполняется неравенство $B>G$, т.е. параметром, ограничивающим механическую стабильность кристаллов, является модуль сдвига.

Рассчитанные для $\mathrm{Nb}_{2} \mathrm{BN}$ и $\mathrm{Mo}_{2} \mathrm{BC}$ упругие модули $B, G$ в целом близки к соответствующим модулям карбидов, нитридов, боридов Мо и Nb. Так, например, модули сжатия боронитрида $(B=282.6 \mathrm{GPa})$ и борокарбида $(B=333.2 \mathrm{GPa})$ сравнимы по величине с $B=283-302 \mathrm{GPa}$ для различных модификаций $\mathrm{NbN}$ [24], $B=299 \mathrm{GPa}$ для $\mathrm{Nb}_{2} \mathrm{~N}_{3} \quad$ [25], $B=262$ и $308 \mathrm{GPa}$ для $\mathrm{NbB}$ и $\mathrm{MoB}$ со структурой $\mathrm{CrB}$ [17], $B=328-341 \mathrm{GPa}$ для монокарбидов молибдена МoC [26], $B=264-285 \mathrm{GPa}$ для МoB со структурами $\mathrm{NiAs}, \mathrm{WC}, \mathrm{NaCl}$ и $\mathrm{CsCl}$ [27] и превышают значения $B=205-238 \mathrm{GPa}$ для $\mathrm{NbB}$ со структурами $\mathrm{NiAs}$ и $\mathrm{NaCl}$ [27]. В свою очередь модули сдвига $\mathrm{Nb}_{2} \mathrm{BN}$ $(G=182.2 \mathrm{GPa})$ и $\mathrm{Mo}_{2} \mathrm{BC}(G=196.8 \mathrm{GPa})$ близки к $G=198$ и $202 \mathrm{GPa}$ для $\mathrm{NbB}, \mathrm{MoB}$ со структурой $\mathrm{CrB}$ [17] и $G=152-224 \mathrm{GPa}$ для полиморфных модификаций МоС [26], но заметно выше значений $G=141 \mathrm{GPa}$ для $\mathrm{Nb}_{2} \mathrm{~N}_{3}$ [25], $G=80-167 \mathrm{GPa}$ для МоB со структурами $\mathrm{NiAs}, \mathrm{WC}, \mathrm{NaCl}$ и $\mathrm{CsCl}[27]$ и $G=83-135 \mathrm{GPa}$ для $\mathrm{NbB}$ со структурами $\mathrm{NiAs}$ и $\mathrm{NaCl}$ [27].

Проанализируем также хрупкопластичное поведение этих материалов. В качестве критерия хрупкопластичного перехода использовался так называемый индикатор Пуга (отношение $G / B$ ) [28]. Согласно этому критерию, материал считается пластичным, если $G / B<0.5$; наоборот, он будет демонстрировать хрупкость, если $G / B>0.5$. Из табл. 6 можно видеть, что $\mathrm{Nb}_{2} \mathrm{BN}$ и $\mathrm{Mo}_{2}$ ВС являются хрупкими, причем хрупкость боронитрида выше, чем борокарбида. Данный факт соответствует результатам оценок для некоторых бинарных карбидов, боридов переходных металлов, таких как $\operatorname{NbB}(G / B=0.76)$ и $\mathrm{MoB}(G / B=0.66)$ со структурой $\mathrm{CrB}$ [17], $\beta$-MoC $(G / B=0.66)$ [26], NbB со структурой $\mathrm{NaCl}(G / B=0.57), \mathrm{MoB}$ со структурами $\mathrm{NaCl}$ $(G / B=0.60)$ и $\mathrm{CsCl}(G / B=0.51)[27]$, но в то же время не совпадает с полученными данными для $\alpha$-МоС $(G / B=0.46)$ и $\gamma$-МoC $(G / B=0.47), \mathrm{NbB}$ со структурой $\mathrm{NiAs}(G / B=0.40)$ [27], МоB со структурами NiAs $(G / B=0.44)$ и WC $(G / B=0.30)$, а также $\mathrm{Nb}_{2} \mathrm{~N}_{3}$ $(G / B=0.47)[25]$, которые являются пластичными. Таким образом, по шкале пластичности $\mathrm{Mo}_{2} \mathrm{BC}$ по сравнению с $\mathrm{Nb}_{2} \mathrm{BN}$ занимает более выгодное положение, а его рассмотрение в качестве перспективного материала для защитных покрытий вполне оправдано (см. [7,8]). Кроме того, относительная хрупкость данных материалов подтверждается также отношением Пуассона v: известно, что для хрупких ковалентных материалов значения $v$ малы, в то время как для пластичных металлических материалов типичное значение $v=0.33$ [29]. 

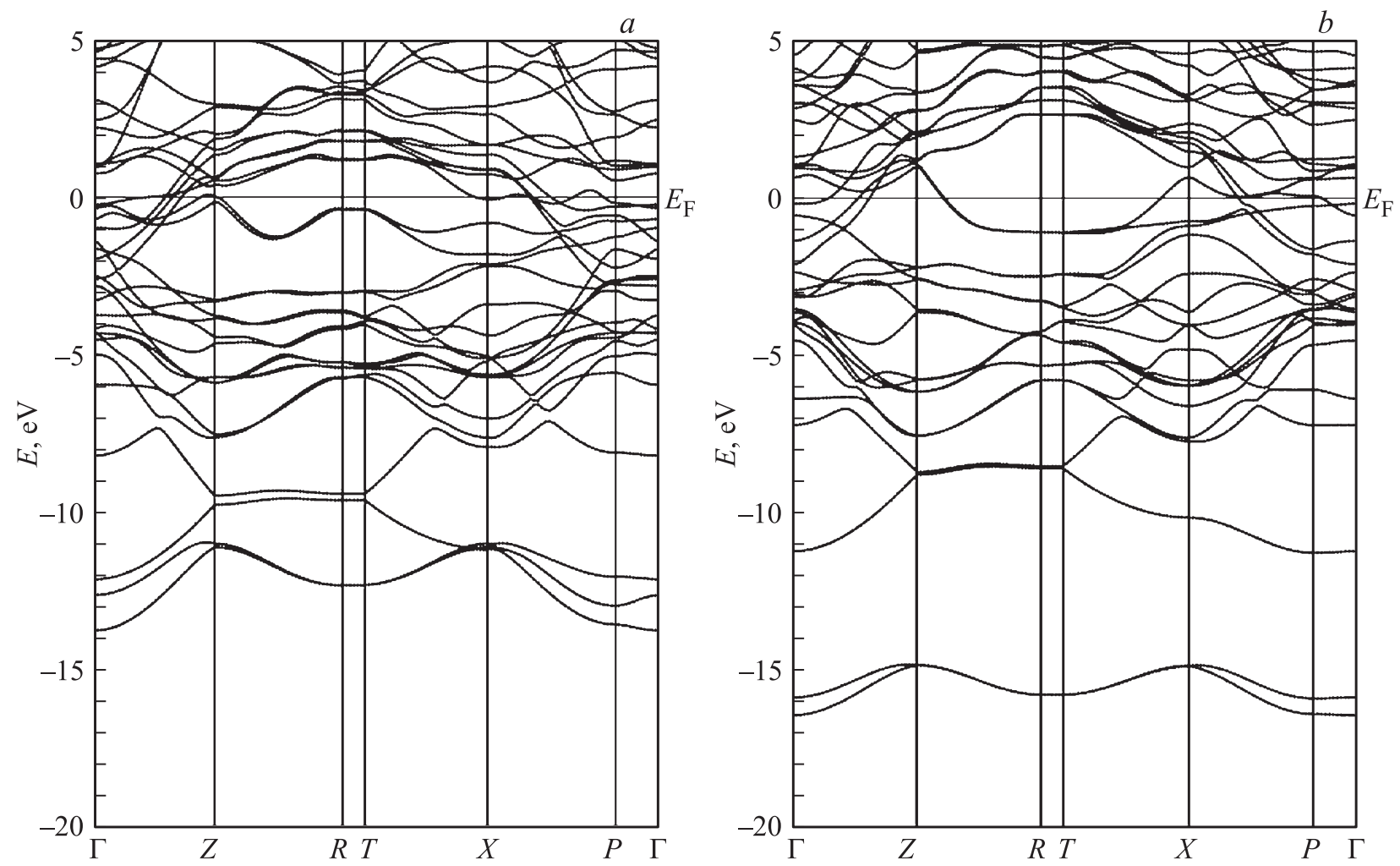

Рис. 2. Рассчитанные зонные структуры вдоль высокосимметричных направлений зоны Бриллюэна для $\mathrm{Mo}_{2} \mathrm{BC}(a)$ и $\mathrm{Nb}_{2} \mathrm{BN}(b)$.

Важнейшей величиной, определяющей практическое использование материала, является его микротвердость. В настоящее время предложен ряд эмпирических соотношений между микротвердостью по Виккерсу $H_{V}$ и упругими модулями [30], однако наиболее реалистичные результаты получаются при использовании в качестве определяющего параметра модуля сдвига $G$ и отношения $k=G / B$. Так, в модели Тяна микротвердость оценивается как $H_{V 1}=0.92 k^{1.137} G^{0.708}$ [31]. Другое выражение было предложено Ченом в работе [32]: $H_{V 2}=2\left(k^{2} G\right)^{0.585}-3$. Из полученных данных видно, что все значения микротвердости $\mathrm{Nb}_{2} \mathrm{BN}$ и $\mathrm{Mo}_{2} \mathrm{BC}$ достаточно близки (табл. 7), при этом значения $H_{V}$ составляют $\sim 20 \mathrm{GPa}$. Кроме того, согласно проведенным оценкам, боронитрид обладает несколько большей микротвердостью. Рассчитанные значения микротвердости для $\mathrm{Mo}_{2} \mathrm{BC}$ хорошо согласуются с результатом экспериментов [11] (табл. 7).

Таблица 7. Рассчитанная микротвердость по Виккерсу $H_{V i}$ (в GPa) в моделях Тяна и Чена $[31,32]$ и среднее значение $H_{V a v}$ для $\mathrm{Nb}_{2} \mathrm{BN}$ и $\mathrm{Mo}_{2} \mathrm{BC}$

\begin{tabular}{c|c|c|c}
\hline Система & $H_{V 1}$ & $H_{V 2}$ & $H_{V \text { av }}$ \\
\hline $\mathrm{Nb}_{2} \mathrm{BN}$ & 22.26 & 22.14 & 22.20 \\
$\mathrm{Mo}_{2} \mathrm{BC}$ & 21.28 & 20.74 & 21.01 \\
Эксперимент & & $20.5 \pm 0.6[11]$ &
\end{tabular}

Далее рассмотрим особенности зонной структуры и плотностей электронных состояний (ПС) $\mathrm{Nb}_{2} \mathrm{BN}$ и $\mathrm{Mo}_{2} \mathrm{BC}$. Основные результаты проведенных зонных расчетов FLAPW-GGA представлены на рис. 2, 3.

Сначала проанализируем электронные свойства $\mathrm{Nb}_{2}$ BN. Из полученных зонных структур и электронных спектров видно, что валентная зона этого соединения состоит из пяти основных полос. Наиболее низкоэнергетическая полоса образована в основном $\mathrm{N} 2 s$-состояниями и располагается в интервале от -16.5 до $-14.8 \mathrm{eV}$ ниже уровня Ферми $\left(E_{\mathrm{F}}\right)$ (пик $\left.A\right)$. Следующая полоса, лежащая в интервале от -11.4 до $-7.7 \mathrm{eV}$ ниже $E_{\mathrm{F}}$, обусловлена в основном В $2 s$-состояниями (область $B$ ). Эти полосы отделены друг от друга запрещенной щелью $\sim 3.4 \mathrm{eV}$. Третья и четвертая полосы, в которых доминируют $\mathrm{Nb} 4 d$-, В $2 p$ - и $\mathrm{N} 2 p$-состояния с добавками В $2 s$ - и $\mathrm{Nb} s, p$-состояний, располагаются в диапазоне от -7.7 до $-1.5 \mathrm{eV}$ ниже $E_{\mathrm{F}}$, перекрываются и отвечают за образование ковалентных связей $\mathrm{Nb}-\mathrm{B}$ и $\mathrm{Nb}-\mathrm{N}$ (пики $C$ и $D$ ). Между этими полосами находится также область гибридизации между В $2 s-$ и В $2 p$-состояниями с центром около $-6 \mathrm{eV}$ ниже $E_{\mathrm{F}}$. Наконец, пятая полоса, непосредственно примыкающая к $E_{\mathrm{F}}$, включает в себя в основном $\mathrm{Nb} 4 d$-состояния с небольшой добавкой $\mathrm{N}$ $2 p$ - и В $2 p$-состояний и отвечает за образование металлических связей $\mathrm{Nb}-\mathrm{Nb}$ между соседними атомами $\mathrm{Nb}$ (пик $E$ ). На уровне Ферми $E_{\mathrm{F}}$ и в нижней части зоны проводимости также доминируют $\mathrm{Nb} 4 d$-состояния. 

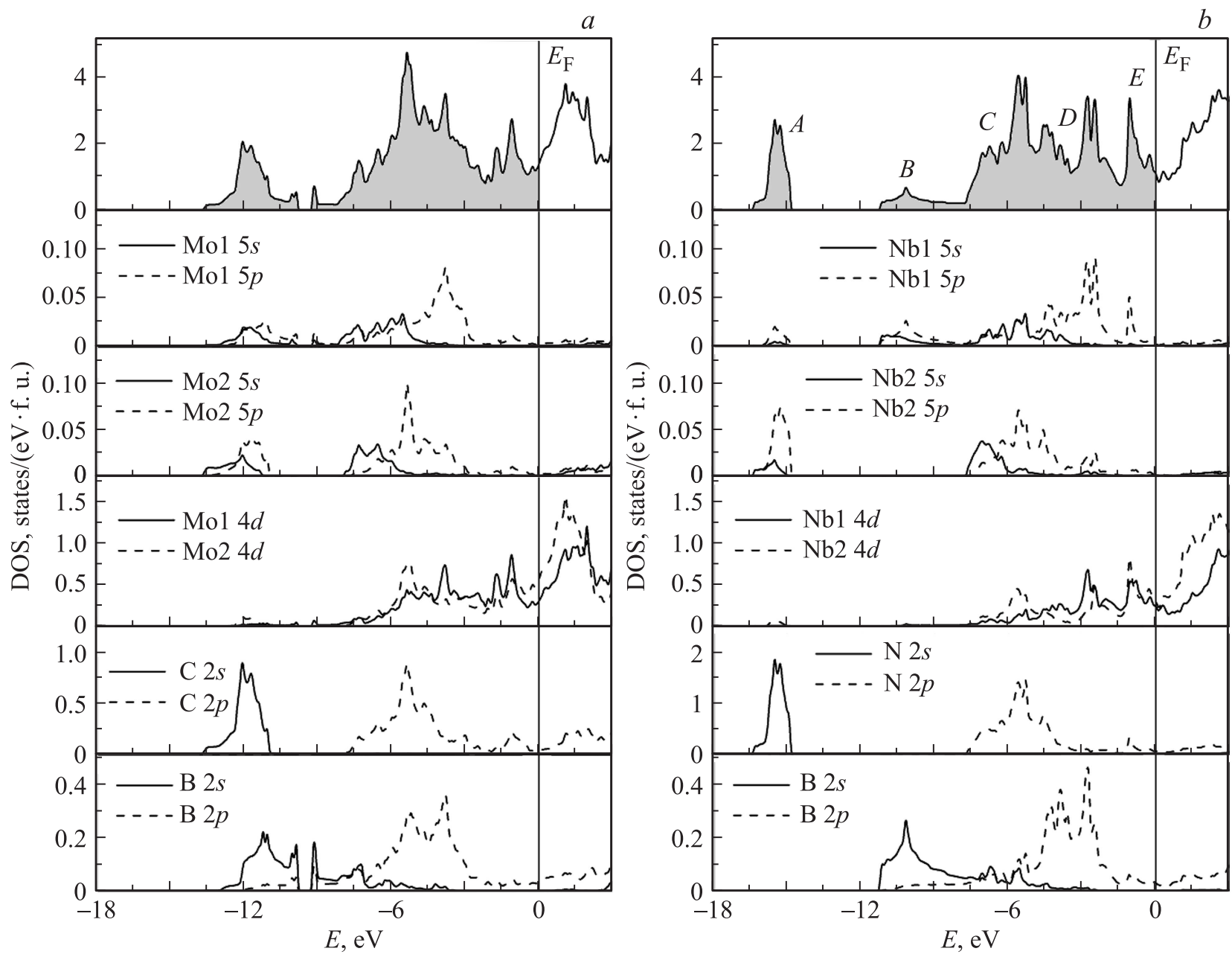

Рис. 3. Полные (вверху) и парциальные плотности электронных состояний для $\mathrm{Mo}_{2} \mathrm{BC}(a)$ и $\mathrm{Nb}_{2} \mathrm{BN}(b)$.

Электронный спектр борокарбида $\mathrm{Mo}_{2} \mathrm{BC}$ в целом схож с таковым для боронитрида, однако имеются и некоторые изменения, состоящие в следующем. Во-первых, нижняя квазиостовная зона существенно уширяется (более чем в 2 раза) до $4.1 \mathrm{eV}$ и образована не только С $2 s$-, но и В $2 s$-состояниями. Во-вторых, значительно уменьшаются как ширина основной валентной зоны, так и ширина энергетической щели между первой и второй зонами, составляющая всего около $0.4 \mathrm{eV}$. Наконец, две перекрывающиеся гибридные полосы располагаются значительно ближе друг к другу. Необходимо отметить, что вид электронного спектра $\mathrm{Mo}_{2} \mathrm{BC}$ в целом совпадает с рассчитанным в работе [7].

Для понимания особенностей электронных свойств $\mathrm{Nb}_{2} \mathrm{BN}$ и $\mathrm{Mo}_{2} \mathrm{BC}$ более подробно проанализируем их околофермиевскую область. Для борокарбида $\mathrm{Mo}_{2} \mathrm{BC}$ $E_{\mathrm{F}}$ попадает в область невысокой ПС (рис. 3). При переходе от $\mathrm{Mo}_{2} \mathrm{BC}$ к $\mathrm{Nb}_{2} \mathrm{BN}$ концентрация валентных электронов уменьшается на один электрон, поэтому следует ожидать, что $E_{\mathrm{F}}$ будет смещаться вниз по шкале энергий и окажется около локального минимума ПС. Действительно, данный эффект хорошо виден из рис. 3.
В результате ПС на уровне Ферми $N\left(E_{\mathrm{F}}\right)$ уменьшается с 1.43 до 1.15 states/(eV·f.u.)

Основной вклад в прифермиевскую область ПС для $\mathrm{Nb}_{2} \mathrm{BN}$ и $\mathrm{Mo}_{2} \mathrm{BC}$ вносят $\mathrm{Nb}(\mathrm{Mo}) 4 d$-состояния $(\sim 50-60 \%)$ с добавлением N $2 p$ - и В $2 p$-состояний, при этом $N\left(E_{\mathrm{F}}\right)$ для металла в позиции 2 выше, чем в позиции 1 (табл. 8). С использованием полученных значений $N\left(E_{\mathrm{F}}\right)$ были также рассчитаны связанные с ними коэффициенты низкотемпературной электронной теплоемкости $\gamma=\left(\pi^{2} / 3\right) N\left(E_{\mathrm{F}}\right) k_{\mathrm{B}}^{2}$ и парамагнитная восприимчивость Паули $\chi=\left(\mu_{\mathrm{B}}\right)^{2} N\left(E_{\mathrm{F}}\right)$ (табл. 8). Видно, что теоретическое значение $\gamma$ сильно занижено относительно экспериментального. Этот факт можно объяснить тем, что экспериментальное значение $\gamma$ было получено для нестехиометрического по азотной подрешетке образца $\mathrm{Nb}_{2} \mathrm{BN}$ (см. [13]). И как мы показали выше (табл. 4), существование такой нестехиометрии (вакансии по подрешетке азота) является энергетически выгодным. Поскольку образование вакансий приводит к уменьшению числа электронов в элементарной ячейке, $E_{\mathrm{F}}$ сдвигается в низкоэнергетическую область в сторону более высокой ПС (рис. 3). Действительно, при экспери- 
Таблица 8. Полные и парциальные плотности электронных состояний на уровне Ферми $N\left(E_{\mathrm{F}}\right), N^{l}\left(E_{\mathrm{F}}\right)$ (в states/(eV · f.u.)), коэффициенты низкотемпературной электронной теплоемкости $\gamma\left(\right.$ в $\left.\mathrm{mJ} /\left(\mathrm{K}^{2} \cdot \mathrm{mol}\right)\right)$ и парамагнитная восприимчивость Паули $\gamma$ (в $10^{-4} \mathrm{emu} / \mathrm{mol}$ ) для $\mathrm{Nb}_{2} \mathrm{BN}$ и $\mathrm{Mo}_{2} \mathrm{BC}$

\begin{tabular}{c|c|c|c|c|c|c|c|c}
\hline Система & $N\left(E_{\mathrm{F}}\right)$ & $N^{M_{1} d}\left(E_{\mathrm{F}}\right)$ & $N^{M_{2} d}\left(E_{\mathrm{F}}\right)$ & $N^{M s, p}\left(E_{\mathrm{F}}\right)$ & $N^{\mathrm{N}(\mathrm{C}) 2 p}\left(E_{\mathrm{F}}\right)$ & $N^{\mathrm{B} 2 p}\left(E_{\mathrm{F}}\right)$ & $\gamma$ & $\chi$ \\
\hline $\mathrm{Nb}_{2} \mathrm{BN}$ & 1.151 & 0.246 & 0.303 & 0.009 & 0.073 & 0.033 & 2.73 & 0.35 \\
& & & & & & & $(6.3[13])$ & \\
$\mathrm{Mo}_{2} \mathrm{BC}$ & 1.436 & 0.313 & 0.573 & 0.009 & 0.051 & 0.037 & 3.40 & 0.44
\end{tabular}

Пр имечание. В скобках приведены данные экспериментальной работы [13].

ментально определенной константе электрон-фононного взаимодействия $\lambda \sim 0.62$ [13], согласно нашим оценкам, проведенным в рамках модели жесткой зоны, теоретические и экспериментальные величины $\gamma$ будут совпадать при концентрации азотных вакансий $x \sim 0.054$, что соответствует эксперименту [13].

Далее на основании самосогласованных расчетов были построены поверхности Ферми (ПФ) комплектных $\mathrm{Mo}_{2} \mathrm{BC}$ и $\mathrm{Nb}_{2} \mathrm{BN}$ и нестехиометрических $\mathrm{Nb}_{2} \mathrm{BN}_{1-x}$ $(x=0.054,0.072)$. Для последнего случая была использована модель жесткой зоны. Эти концентрации нестехиометрии были выбраны следующим образом: для $\mathrm{Nb}_{2} \mathrm{BN}_{1-x}$ с $x=0.054$ совпадают теоретическое и экспериментальное значения коэффициентов Зоммерфельда $\chi^{\text {theor }}=\gamma^{\text {exp }}$, а в случае $x=0.072 E_{\mathrm{F}}$ попадает на интенсивный пик ПС. Вид всех ПФ показан на рис. 4.

Обсудим сначала топологию ПФ для комплектных $\mathrm{Mo}_{2} \mathrm{BC}$ и $\mathrm{Nb}_{2} \mathrm{BN}$. Отметим, что полученная нами ПФ для $\mathrm{Mo}_{2} \mathrm{BC}$ полностью соответствует результату других теоретических расчетов [33]. ПФ $\mathrm{Mo}_{2} \mathrm{BC}$ состоит из пяти листов, четыре из которых электронного типа и один дырочного. Два электронных листа представлены квазиэллиптическими поверхностями, центрированными около точки Г. Третий электронный лист состоит из еще одной эллиптической поверхности с добавлением небольших боковых карманов. Четвертый лист электронного типа образован четырьмя симметрично расположенными карманами и вытянутой вдоль оси $z$ замкнутой поверхностью. Наконец, пятый дырочный лист образуют дополнительные небольшие карманы, расположенные сверху и снизу вдоль оси $z$ в зоне Бриллюэна, (рис. 4,a).

Для стехиометрического боронитрида $\mathrm{Nb}_{2} \mathrm{BN}$ поверхность Ферми представлена четырьмя листами: тремя листами электронного типа и одним дырочного. Три электронных листа сформированы двумя небольшими искаженными параболическими цилиндрами вверху (внизу) зоны Бриллюэна, центрированными относительно точки $Z$, эллипсоидом с двумя перемычками посередине около точки $\Gamma$, а также четырьмя небольшими „рогообразными“ карманами (рис. 4, $b$ ). Четвертый лист дырочного типа состоит из незамкнутых цилиндрических поверхностей в боковой части зоны Бриллюэна совместно с $w$-образными поверхностями, расположенными вверху (внизу) зоны Бриллюэна.
При нестехиометрии по азотной подрешетке в целом топология четырех характеристических листов ПФ сохраняется (рис. $4, c, d$ ). Основное изменение состоит в следующем: две поверхности, принадлежащие четвертому листу дырочного типа, соединяются друг с другом. При этом $w$-образная поверхность, содержащая в случае $\mathrm{Nb}_{2} \mathrm{BN}$ близкие к плоским участки, становится более гладкой. Одновременно претерпевает незначительные изменения эллипсоид, а также различные виды карма-

$a$

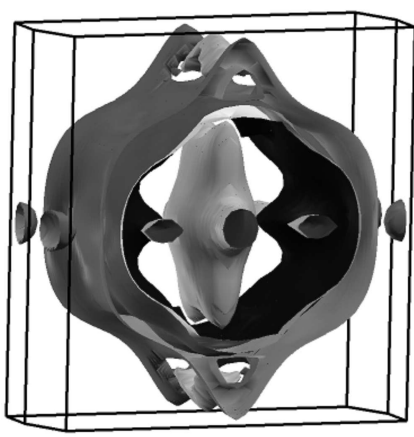

$b$
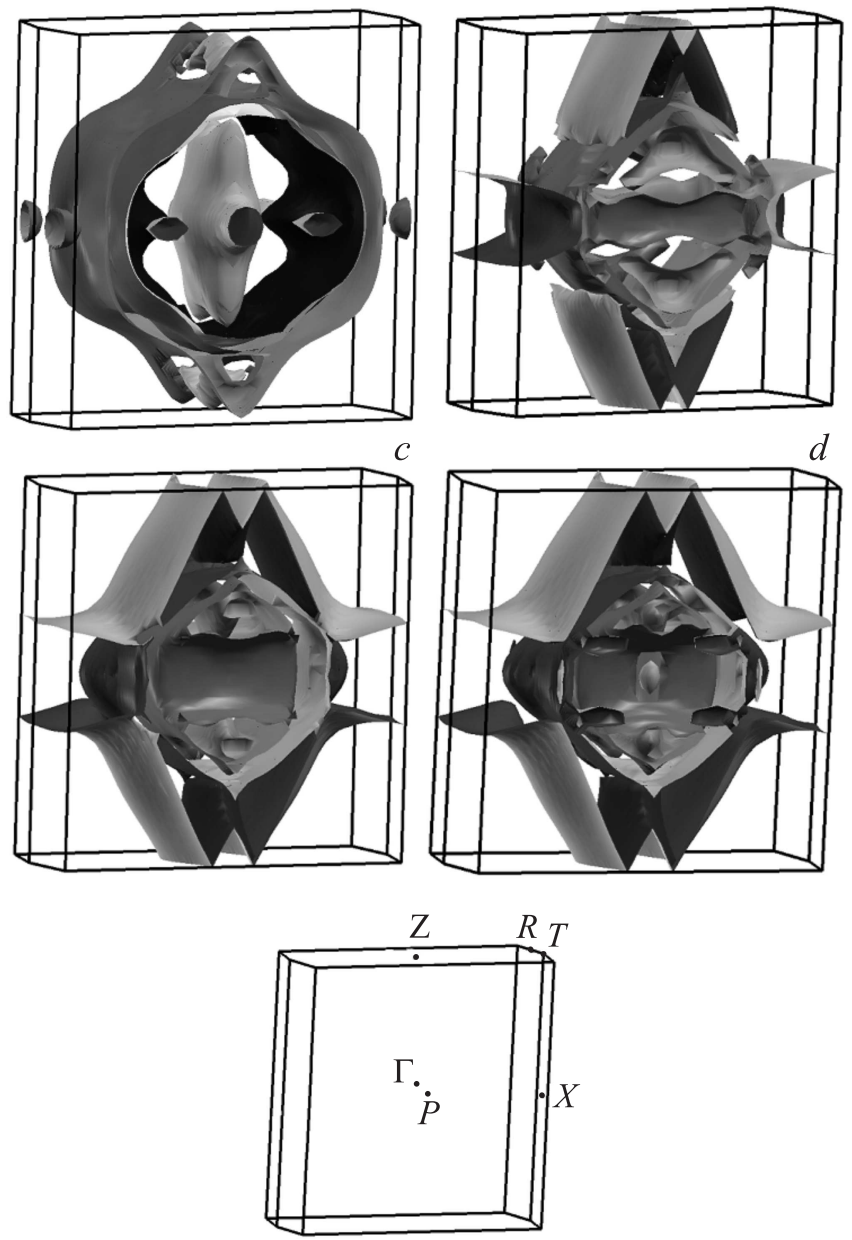

Рис. 4. Рассчитанные поверхности Ферми для $\mathrm{Mo}_{2} \mathrm{BC}(a)$, $\mathrm{Nb}_{2} \mathrm{BN}(b)$ и $\mathrm{Nb}_{2} \mathrm{BN}_{1-x}$ с $x=0.054(c)$ и $0.072(d)$. Внизу приведена зона Бриллюэна с указанием характерных точек. 

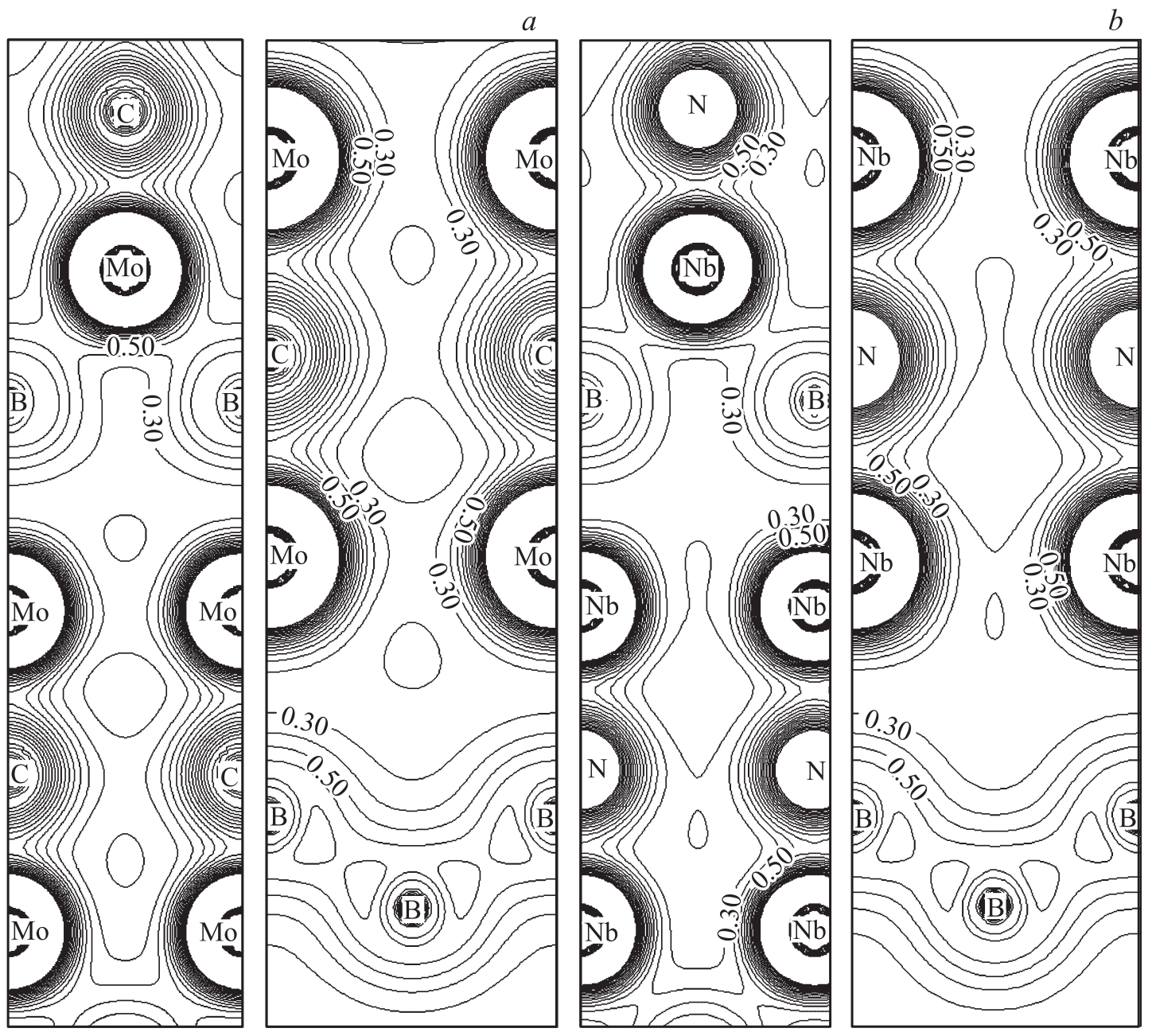

Рис. 5. Распределение зарядовой плотности валентных состояний в плоскостях $(100)$ и $(001)$ для $\mathrm{Mo}_{2} \mathrm{BC}(a)$ и $\mathrm{Nb}_{2} \mathrm{BN}(b)$. Разность между изоэлектронными контурами составляет $0.1 \mathrm{e} / \AA^{3}$.

нов. Так, например, „рогообразные“ карманы переходят в эллиптические (рис. 4).

Межатомные взаимодействия в $\mathrm{Nb}_{2} \mathrm{BN}$ и $\mathrm{Mo}_{2} \mathrm{BC}$ могут быть представлены как сочетание трех компонент: ковалентной, ионной и металлической. Ковалентная составляющая в данных системах имеет место вследствие наличия гибридизации между $\mathrm{Nb}(\mathrm{Mo}) 4 d$-состояниями и $\mathrm{N}(\mathrm{C}) 2 p$-состояниями, а также В $2 p$-состояниями (рис. 3) и подтверждается присутствием заметных направленных связей $\mathrm{Nb}(\mathrm{Mo})-\mathrm{N}(\mathrm{C})$ и $\mathrm{Nb}(\mathrm{Mo})-\mathrm{B}$ на со- ответствующих картах зарядовой плотности валентных состояний (рис. 5). Атомы $\mathrm{Nb}(\mathrm{Mo})$, находящиеся в двух неэквивалентных позициях, образуют ковалентные связи с $\mathrm{N}(\mathrm{C})$ одновременно, в то время как с В будет взаимодействовать главным образом металл, находящийся в позиции 1. Кроме того, из рис. 3 видна еще и В $2 s-\mathrm{B} 2 p$-гибридизация, отвечающая за образование ковалентных связей $\mathrm{B}-\mathrm{B}$ в цепочках атомов (рис. 5). Прямые связи $\mathrm{N}-\mathrm{N}$ и $\mathrm{C}-\mathrm{C}$ в $\mathrm{Nb}_{2} \mathrm{BN}$ и $\mathrm{Mo}_{2} \mathrm{BC}$ отсутствуют.

Таблица 9. Атомные заряды в модели Бейдера $Q$ (в $e$ ) и атомная зарядовая разность в сравнении с нейтральными атомами $\Delta Q=Z-Q($ в $e)$ для боронитрида $\mathrm{Nb}_{2} \mathrm{BN}$ и борокарбида $\mathrm{Mo}_{2} \mathrm{BC}$

\begin{tabular}{c|c|c|c|c|c|c|c|c}
\hline \multirow{2}{*}{ Система } & \multicolumn{2}{|c|}{$M_{1}$} & \multicolumn{2}{c|}{$M_{2}$} & \multicolumn{2}{c}{ В } & \multicolumn{2}{c}{$\mathrm{N}(\mathrm{C})$} \\
\cline { 2 - 9 } & $Q$ & $\Delta Q$ & $Q$ & $\Delta Q$ & $Q$ & $\Delta Q$ & $Q$ & $\Delta Q$ \\
\hline $\mathrm{Nb}_{2} \mathrm{BN}$ & 39.901 & +1.099 & 39.480 & +1.522 & 5.943 & -0.943 & 8.672 & -1.672 \\
$\mathrm{Mo}_{2} \mathrm{BC}$ & 41.183 & +0.817 & 40.720 & +1.280 & 5.671 & -0.671 & 7.423 & -1.423
\end{tabular}


Ионная составляющая обусловлена имеющейся разницей в электроотрицательности атомов $\mathrm{Nb}(\mathrm{Mo}), \mathrm{N}(\mathrm{C})$ и В и возникающим из-за этого частичным переносом заряда с атомов $4 d$-металла на атомы азота (углерода) и бора. Для оценки ионной компоненты мы также рассчитали атомные заряды по схеме Бейдера [34] (табл. 9). Исходя из полученных данных, перенос заряда на атомы В и $\mathrm{N}(\mathrm{C})$ играет существенную роль в данных соединениях, так как атомные заряды $Q$ имеют промежуточные значения между зарядами на нейтральных атомах и зарядами в чисто ионной модели. Кроме того, обращает на себя внимание заметное различие между зарядами на атомах $4 d$-металла в позициях 1 и 2 , при этом $Q\left(M_{1}\right)>Q\left(M_{2}\right)$ (табл 9).

Наконец, металлическая компонента межатомных взаимодействий обусловлена делокализованными $\mathrm{Nb}(\mathrm{Mo})$ $4 d$-состояниями и включает в себя взаимодействия типа металл-металл, которые видны из рис. 5. Несколько более сильные межатомные взаимодействия для $\mathrm{Mo}_{2} \mathrm{BC}$ в сравнении с $\mathrm{Nb}_{2} \mathrm{BN}$ в силу меньшей энергии когезии (см. выше) объясняются уменьшением всех основных межатомных расстояний $M_{1}-\mathrm{N}(\mathrm{C}), M_{2}-\mathrm{N}(\mathrm{C}), M_{1}-\mathrm{B}$, $M_{1}-M_{2}$ и В $-\mathrm{B}$ (табл. 3).

\section{4. Заключение}

В настоящей работе с использованием $a b$ initio зонного метода FLAPW-GGA впервые проведено сравнительное исследование электронной структуры, поверхностей Ферми, энергий когезии, формирования фаз и точечных дефектов, механических свойств и межатомных взаимодействий боронитрида $\mathrm{Nb}_{2} \mathrm{BN}$ и борокарбида $\mathrm{Mo}_{2} \mathrm{BC}$.

Согласно нашим оценкам, энергии формирования $\mathrm{Mo}_{2} \mathrm{BC}$ и $\mathrm{Nb}_{2} \mathrm{BN}$ отрицательны, т. е. эти соединения являются термодинамически стабильными по отношению к простым элементам. При этом боронитрид более стабилен, чем борокарбид. Энергии образования вакансий по подрешеткам азота и углерода в $\mathrm{Nb}_{2} \mathrm{BN}$ и $\mathrm{Mo}_{2} \mathrm{BC}$ отрицательны, что свидетельствует об энергетической выгодности существования этих вакансий.

$\mathrm{Mo}_{2} \mathrm{BC}$ и $\mathrm{Nb}_{2} \mathrm{BN}$ удовлетворяют критерию механической стабильности кристаллов. Для них выполняется соотношение $B>G$, и параметром, ограничивающим стабильность систем, является модуль сдвига. Модули сжатия и сдвига $\mathrm{Mo}_{2} \mathrm{BC}, \mathrm{Nb}_{2} \mathrm{BN}$ сравнимы по величине с модулями родственных бинарных карбидов, нитридов, боридов. Максимальными значениями всех упругих модулей обладает $\mathrm{Mo}_{2} \mathrm{BC}$; анализ данных систем по шкале хрупкость/пластичность показал, что $\mathrm{Mo}_{2} \mathrm{BC}$ и $\mathrm{Nb}_{2} \mathrm{BN}$ являются хрупкими материалами, но первый является более пластичным. Проведенные расчеты микротвердости по Виккерсу $H_{V}$ с использованием двух эмпирических формул показывают, что изучаемые системы относятся к материалам со средней твердостью $\left(H_{V} \sim 20 \mathrm{GPa}\right)$.
Из анализа электронных спектров этих соединений было установлено, что в прифермиевской области доминируют $4 d$-состояния металла, а вклад атомов $\mathrm{Nb}(\mathrm{Mo})$ в позиции 2 существенно выше, чем в позиции 1. При переходе от $\mathrm{Mo}_{2} \mathrm{BC}$ к $\mathrm{Nb}_{2} \mathrm{BN}$ вследствие уменьшения концентрации валентных электронов происходит сдвиг уровня Ферми вниз по шкале энергий, в результате он попадает в локальный минимум ПС. Поэтому имеем ПС на уровне Ферми $N\left(E_{\mathrm{F}}\right)\left(\mathrm{Mo}_{2} \mathrm{BC}\right)>N\left(E_{\mathrm{F}}\right)\left(\mathrm{Nb}_{2} \mathrm{BN}\right)$. Высказано предположение, что существенное различие значений теоретического и экспериментального коэффициентов Зоммерфельда $\gamma$ обусловлено наличием азотных вакансий в $\mathrm{Nb}_{2} \mathrm{BN}$, существование которых установлено в эксперименте и в наших расчетах. Определена топология поверхностей Ферми стехиометрического боронитрида $\mathrm{Nb}_{2} \mathrm{BN}$ и боронитрида, содержащего вакансии по подрешетке азота с различной концентрацией $x$, полученная в модели жесткой зоны. Установлено, что количество листов и их вид для $\mathrm{Nb}_{2} \mathrm{BN}_{1-x}$ в целом остается неизменным вне зависимости от параметра $x$. Межатомные взаимодействия в $\mathrm{Nb}_{2} \mathrm{BN}$ и $\mathrm{Mo}_{2} \mathrm{BC}$ имеют сложный металлически-ионно-ковалентный тип и представляют разнообразие соответствующих компонент. Оценки зарядов $Q$ на атомах по схеме Бейдера указывают на то, что они принимают промежуточные значения между зарядами нейтральных атомов и зарядами, получаемыми для чисто ионной модели.

\section{Список литературы}

[1] Л. Тот. Карбиды и нитриды переходных металлов. Мир, М. (1974). 294 c.

[2] Г.В. Самсонов, И.М. Винницкий. Тугоплавкие соединения. Металлургия, М. (1976). 560 с.

[3] Дж. Гольшмидт. Сплавы внедрения. Мир, М. (1971). Т. 1. $424 \mathrm{c}$

[4] Г.В. Самсонов, Л.Я. Марковский, А.Ф. Жигач, М.Г. Валяшко. Бор, его соединения и сплавы. Изд-во АН СССР, Киев (1960). 590 c.

[5] E.L. Muetterties. The chemistry of boron and its compounds. Wiley, N. Y. (1967). 699 p.

[6] Г.В. Самсонов, Т.И. Серебрякова, В.А. Неронов. Бориды. Атомиздат, М. (1975). 376 с.

[7] J. Emmerlich, D. Music, M. Braun, P. Fayek, F. Munnik, J.M. Schneider. J. Phys. D 42, 185406 (2009).

[8] H. Bolvardi, J. Emmerlich, M. to Baben, D. Music, J. von Appen, R. Dronskowski, J.M. Schneider. J. Phys.: Condens. Matter. 25, 045501 (2013).

[9] W. Jeitschko, H. Nowotny, F. Benesovsky. Monatsh. Chem. 94, 565 (1963).

[10] L.E. Toth. J. Less-Common Met. 13, 129 (1967).

[11] P. Lejay, B. Chevalier, J. Etourneau, P. Hagenmuller, P. Peshev. Synth. Met. 4, 139 (1981).

[12] G.P. Rogl. Phase diagrams of ternary metal-boron-carbon systems. MSIT-ASM International (1998). $261 \mathrm{p}$.

[13] O.V. Cigarroa, S.T. Renosto, A.J.S. Machado. Supercond. Sci. Technol. 27, 035005 (2014). 
[14] P. Blaha, K. Schwarz, G.K.H. Madsen, D. Kvasnicka, J. Luitz. WIEN2k. An augmented plane wave plus local orbitals program for calculating crystal properties. Vienna University of Technology, Vienna (2001). 189 p.

[15] J.P. Perdew, S. Burke, M. Ernzerhof. Phys. Rev. Lett. 77, 3865 (1996).

[16] P.E. Blöchl, O. Jepsen, O.K. Anderson. Phys. Rev. B 49, 16223 (1994).

[17] X. Xu, K. Fu, L. Li, Z. Lu, X. Zhang, Y. Fan, J. Lin, G. Liu, H. Luo, C. Tang. Physica B 419, 105 (2013).

[18] А.И. Гусев, А.А. Ремпель. Нестехиометрия, беспорядок и порядок в твердом теле. Изд-во УрО РАН, Екатеринбург (2001). $580 \mathrm{c}$.

[19] C.G. Van de Walle, J. Neugebauer. J. Appl. Phys. 95, 3851 (2004).

[20] Z.-J. Wu, E.-J. Zhao, H.-P. Xiang, X.-F. Hao, X.-J. Liu, J. Meng. Phys. Rev. B 76, 054115 (2005).

[21] W. Voigt. Lehrbuch der Kristallphysik. Teubner, Leipzig (1928). 962 p.

[22] A. Reuss, Z. Angew. Math. Mech. 9, 49 (1929).

[23] R. Hill. Proc. Phys. Soc. Lond. 65, 350 (1952).

[24] V.I. Ivaschenko, P.E.A. Turchi, E.I. Olifan. Phys. Rev. B 82, 054109 (2010)

[25] J.-D. Zhang, X.-Y. Huang. Physica B 456, 5 (2015).

[26] Y.Z. Liu, Y.H. Jiang, J. Feng, R. Zhou. Physica B 419, 45 (2013).

[27] Y. Wang, W. Chen, X. Chen, H.Y. Liu, Z.H. Ding, Y.M. Ma, X.D. Wang, Q.P. Cao, J.Z. Jiang. J. Alloys Compd. 538, 115 (2012).

[28] S.F. Pugh. Phil. Mag. 45, 823 (1953).

[29] J. Haines, J.M. Leger, G. Bocquillon. Ann. Rev. Mater. Res. 31, 1 (2001)

[30] A.L. Ivanovskii. Int. J. Refract. Met. Mater. 36, 179 (2013).

[31] Y.J. Tian, B. Xu, Z.S. Zhao. Int. J. Refract. Met. Hard Mater. 33, 93 (2012).

[32] X.-Q. Chen, H. Niu, D. Li, Y. Li. Intermetallics 19, 1275 (2011).

[33] R. Falconi, P. de la Mora, F. Morales, R. Escamilla, C.O. Camacho, M. Acosta, R. Escudero, J. Low Temp. Phys. 179, 158 (2015).

[34] R.F.W. Bader. Atoms in molecules: a quantum theory. International series of monographs on chemistry. Clarendon Press, Oxford (1990). 458 p. 